\title{
Salida a un espacio natural para desarrollar competencias interdisciplinares de maestros en formación
}

\author{
Carlos MARTÍNEZ-HERNÁNDEZ \\ María Isabel CIFO-IZQUIERDO \\ Pablo FARINÓS-CELDRÁN \\ Francisco Javier ROBLES-MORAL
}

Datos de contacto:

Carlos Martínez-Hernández Universidad Complutense de Madrid, Departamento de Didáctica de Ciencias Experimentales, Ciencias Sociales y Matemáticas cmartinezhernandez@ucm.es

María Isabel Cifo-Izquierdo Universidad de Murcia, Departamento de Expresión Plástica, Musical y Dinámica mariaisabel.cifo@um.es

Pablo Farinós-Celdrán ISEN Centro Universitario, Área de Didáctica de Ciencias Experimentales pfarinos@um.es

Francisco Javier Robles-Moral Universidad de Murcia, Departamento de Didáctica de las Ciencias Experimentales franciscojavier.moral@um.es

Recibido: 29/04/2021

Aceptado: 09/09/2021

\section{RESUMEN}

Desde el marco constructivista del aprendizaje, basado en métodos activos para el desarrollo de competencias, se presenta una práctica docente efectuada en un espacio natural con maestros en formación, desde las áreas de las Didácticas de Ciencias Sociales, Ciencias Experimentales y Educación Física. El objetivo es cuantificar y analizar el nivel de logro de competencias adquiridas y la valoración didáctica y logística de los participantes. Se recurre a un cuestionario de evaluación y valoración y a una adaptación de la técnica del tendero de los deseos. Los datos, siguiendo una metodología mixta, se analizan cualitativamente y con estadística descriptiva e inferencial, para obtener frecuencias y averiguar si hay diferencias significativas con las calificaciones de clase. Destaca un elevado aprendizaje vivencial, que ha facilitado un aprendizaje trasversal también considerable pero un aprendizaje específico variable según el área de conocimiento, interpretado como resultado de una distinta percepción entre la teoría de clase y la realidad del medio. Así, el desarrollo de competencias ha sido más evidente en Ciencias Sociales (78\% del máximo evaluado y sin diferencias significativas con las calificaciones medias de clase), frente a, en el otro extremo, las competencias propias de Educación Física, de menor adquisición significativa que los valores de calificación de clase. Se concluye que las salidas requieren cierta asiduidad para el desarrollo de competencias percibidas como ajenas al medio, especialmente desde un punto de vista interdisciplinar.

PALABRAS CLAVE: salida didáctica; práctica docente; competencias; Ciencias Sociales; Ciencias Experimentales; Educación Física. 


\title{
Didactic fieldtrip to a natural area for the development of interdisciplinary competencies of teachers in training
}

\begin{abstract}
This paper presents a teaching practice carried out in a natural area with teachers in training, from the areas of Didactics of Social Sciences, Experimental Sciences and Physical Education. The constructivist framework of learning, based on active methods for the development of competencies is followed. The objective is to quantify and analyze the level of achievement of acquired competencies and the didactic and logistical assessment of the participants. An evaluation and assessment questionnaire and an adaptation of the technique of the shopkeeper of wishes are used. Data, following a mixed methodology, are qualitatively analyzed and descriptive and inferential statistically processed to obtain frequencies and find out if there are significant differences with class qualifications. A high experiential learning stands out, which has facilitated a considerable transversal learning but a variable specific learning according to the area of knowledge. This is interpretated as a result of a different perception between the classroom theory and the reality of the environment. The development of competencies has been more evident in Social Sciences $78 \%$ of the maximum evaluated and without significant differences with the average class qualifications). In the other side, the competencies of Physical Education have been developed with less significant values than class qualifications. It is concluded that fieldtrips require a certain assiduity for the development of competencies perceived as alien to the environment, especially from an interdisciplinary point of view.
\end{abstract}

KEYWORDS: fieldtrip; teaching practice; competences; Social Sciences; Experimental Sciences; Physical Education.

\section{Introducción}

\section{Las salidas didácticas en el aprendizaje competencial}

En el marco del Espacio Europeo de Educación Superior (EEES) se diseñan titulaciones basadas en la adquisición de competencias. Adquirirlas supone lograr los conocimientos, las destrezas y las actitudes necesarias para adaptarse y dar respuesta a toda situación que lo requiera, independientemente del contexto (Amor y SerranoRodríguez, 2019). Se debe garantizar una formación en la que se integren todas las competencias (genéricas, específicas y transversales) para alcanzar el desarrollo integral y posibilitar la inserción laboral de los titulados (Asún et al., 2013).

Esto ha supuesto un cambio de paradigma hacia metodologías activas en el que el alumnado se convierte en protagonista para la adquisición de las competencias profesionales (López Facal, 2013; López-Pastor, 2011; Mogensen y Schnak, 2010). Los procesos de innovación marcan el camino para posibilitar este cambio desde la 
calidad docente (Cano et al., 2018; Souto González, 2011), a través de buenas prácticas competenciales (Gabarda, 2015).

Se propone un aprendizaje dialógico, en una continua confrontación con los demás (Freire, 1990). Sin embargo, en la educación superior aún sigue predominando el aprendizaje tradicional de clases magistrales (Rodríguez y Santana, 2015). Para la construcción de un aprendizaje basado en la adquisición de competencias es fundamental la acción didáctica (Martínez et al., 2012), planificada en buenas prácticas.

Una salida didáctica puede ser un buen ejemplo de buenas prácticas por sus posibilidades competenciales, a partir de su definición como una actividad grupal de trabajo de campo con fines didácticos que tiene lugar fuera del aula, en un espacio geográfico que es fuente de conocimiento y al que accede el alumnado a través de una experiencia vivencial de motivación y desarrollo personal (Martínez-Hernández et al., 2021).

Según Crespo et al. (2018), las salidas facilitan la comprensión holística del medio, favoreciendo la concepción multicausal de procesos y elementos espaciales. Esta circunstancia las convierte en una estrategia de probada eficacia en la didáctica de distintas disciplinas, como las Ciencias Sociales (Moreno-Vera y Monteagudo Fernández, 2019; Prats Cuevas, 2001; Sousa Fernandes et al., 2016) y las Ciencias Naturales (Farmer et al., 2007), así como para un aprendizaje interdisciplinar (Benejam, 2003). El Manifiesto por el Aprendizaje Fuera del Aula (Council for Learning Outside the Classroom, 2006) destaca que cualquier joven debería experimentar el mundo que hay más allá del aula, como una parte esencial del aprendizaje y del desarrollo personal. El contacto con el medio natural puede favorecer la salud mental, emocional y social (Maller, 2009; Stern et al., 2008). Los beneficios, como señalan Martínez-Hernández y Yubero (2020), también se han demostrado para espacios urbanos e incluso espacios cerrados, como museos y similares.

A pesar de su largo recorrido histórico (Aguilera, 2018), Pedrinaci (2012) señala que su número sigue siendo relativamente bajo, lo que produce en el estudiante la sensación de novedad. Rebelo et al. (2011) lo atribuyen principalmente a clases masificadas y la dedicación y consabida responsabilidad civil que conllevan. Solbes (2011) señala que las salidas mejoran la percepción de las asignaturas, que muchos estudiantes consideran a priori poco atractivas y desconectadas. Albaladejo y López (2020) actualizan estas consideraciones, lamentando que el conocimiento del entorno siga haciéndose hacia adentro y no hacia afuera.

En el ámbito de la formación del profesorado, Costillo et al. (2014) argumentan que las salidas didácticas no solo benefician el plano afectivo, sino también el cognitivo y las competencias transversales, aumentando la calidad de la educación. Por ello, si los futuros profesores las realizan, pueden ver acrecentado su aprendizaje del medio natural, social y cultural, y, debido a un efecto cascada, también sus futuros alumnos. Peasland et al. (2019) apuntan que la educación superior debe incluir más formación en trabajos de campo. Domínguez Almansa y López Facal (2014) demuestran sus beneficios didácticos en el desarrollo de competencias de maestros en formación y alumnado de educación primaria. 


\section{Planteamiento y objetivos}

Las salidas fuera del aula se constituyen como una estrategia didáctica de reconocida eficacia para el desarrollo de competencias ligadas a múltiples disciplinas, aunque las experiencias en este sentido interdisciplinar, fundamentales en el aprendizaje por competencias (Zabalza, 2006), no son tan abundantes (Domínguez Almansa y López Facal, 2014). Este nuevo paradigma supone una orientación a la coordinación entre el profesorado (Arroyo et al., 2020). Surgen así propuestas educativas abordadas de forma conjunta entre diversas materias (interdisciplinares), con beneficios como la implicación activa de alumnos y docentes, la integración de los conocimientos y competencias que comparten las diferentes asignaturas que intervengan (Lavega et al., 2013), o capacidades esenciales como colaboración y comunicación (Redecker y Punie, 2013). Se trata de beneficios competenciales que muchos autores atribuyen a las salidas didácticas (Aguilera, 2018).

A partir de estas consideraciones, se presenta una propuesta docente basada en una salida interdisciplinar a un espacio natural protegido y dirigida a maestros en formación. Tomando en consideración el paradigma actual del aprendizaje basado en competencias y el marco teórico acerca de las salidas didácticas, así como una metodología constructivista activa, de búsqueda de un aprendizaje significativo e interdisciplinar (Arroyo et al., 2020), la propuesta persigue desarrollar en el alumnado una serie de competencias definidas por las áreas de conocimiento de las Didácticas de las Ciencias Sociales, las Ciencias Experimentales y la Educación Física (en adelante, CC.SS, CC.EE y EF, respectivamente), las cuales son propiciadas por el aprovechamiento del entorno natural en que se desenvuelve la propuesta. El primer objetivo de la investigación es cuantificar y analizar el grado de aprendizaje adquirido por el alumnado tras la implementación de la propuesta. El segundo objetivo es cuantificar y analizar la valoración del alumnado sobre la eficacia didáctica y organizativa de la propuesta docente.

\section{Método}

\section{Área de estudio y participantes}

El espacio natural en el que se ha desarrollado la propuesta docente es el Parque Regional de Sierra Espuña, en el interior de la Región de Murcia (sureste de España); concretamente en las instalaciones de la Finca Caruana (figura 1). El paisaje es de tipo mediterráneo litoral con rasgos de media montaña. Su localización dentro de un espacio natural protegido por la legislación autonómica (Decreto 13/95) ayuda a la conservación de sus elementos naturales y antrópicos. 


\section{Figura 1}

Finca Caruana: localización dentro del espacio natural de Sierra Espuña (izquierda) y plano de instalaciones (derecha)

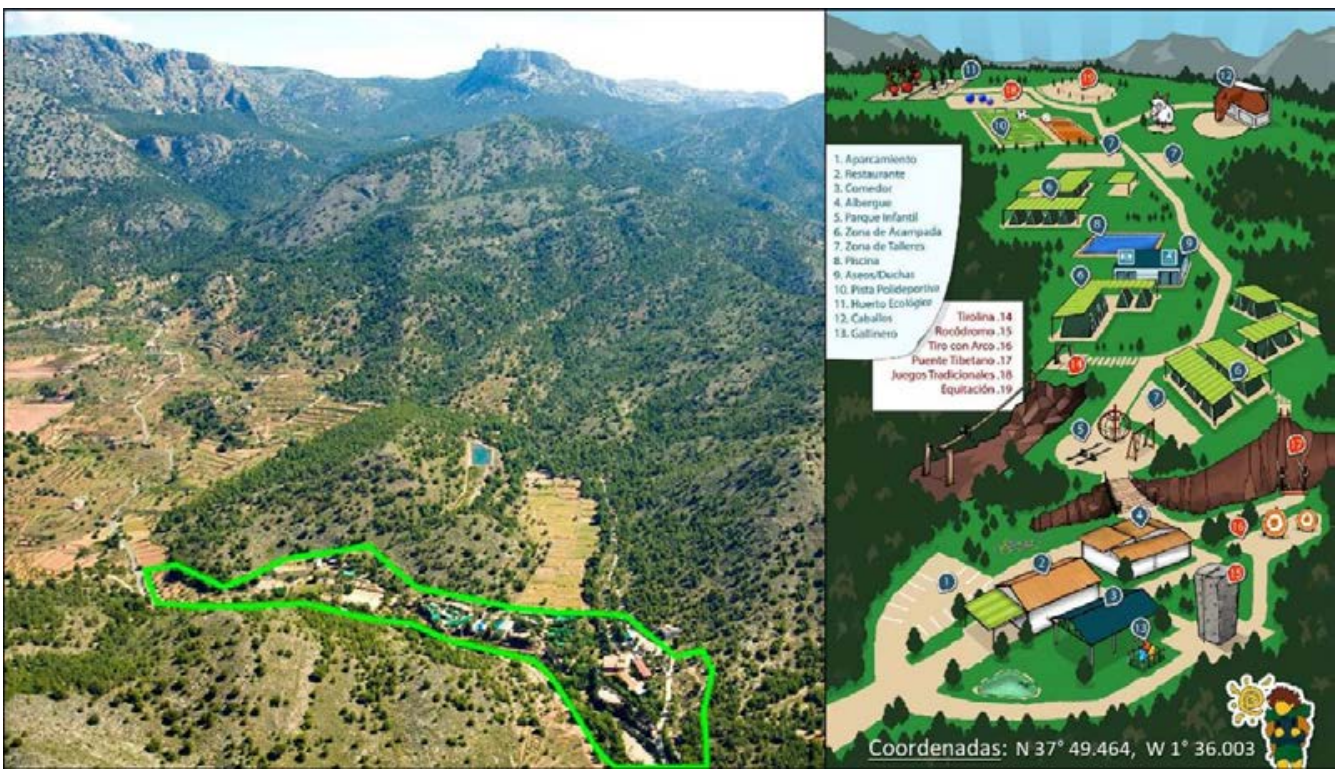

Fuente: www.fincacaruana.es (adaptación)

Los participantes fueron estudiantes de tercer curso del Grado en Educación Primaria de ISEN, una titulación de la Facultad de Educación de la Universidad de Murcia en su centro adscrito de Cartagena (España). En total eran 29 estudiantes (13 chicos y 16 chicas), de edades comprendidas entre los 19 y los 24 años $(M=21, \mathrm{SD}=$ 1.3). El $100 \%$ conforma la muestra de estudio de esta investigación.

\section{Diseño metodológico y curricular}

La propuesta docente se presentó en forma de taller formativo complementario, de 13.5 horas de duración y equivalente a 0.5 créditos CRAU, dentro de la oferta educativa oficial del servicio de Estudios Propios de la Universidad de Murcia, bajo el título de "Taller Trabajo didáctico fuera del aula: Proyecto interdisciplinar en la naturaleza". Tuvo una duración de dos días, por lo que hubo pernoctación, en el albergue de la Finca. Al alumnado se le proporcionó un Dossier de Actividades con toda la información logística y académica.

Se estableció una estructura en 5 bloques, uno por cada una de las tres áreas de conocimiento involucradas, uno de aplicación conjunta y un bloque extra de didáctica general sobre dinámicas de grupo. Estos bloques desarrollan contenidos curriculares y competencias procedentes de materias vinculadas a las citadas áreas de conocimiento en el Grado de Educación Primaria, en relación con el medio natural. Los bloques, a su vez, se relacionaron con "Proyectos" (Tabla 1). 


\section{Tabla 1}

Relación curricular de cada Proyecto de la propuesta docente

\begin{tabular}{|c|c|c|}
\hline Proyecto y descripción & $\begin{array}{l}\text { Materias* } \\
\text { (Comp.) }\end{array}$ & Contenidos \\
\hline \multirow[t]{2}{*}{$\begin{array}{l}\text { I. Sentidos: Familiarización con el medio } \\
\text { natural. } \\
\text { Juegos de presentación y juegos motores en } \\
\text { el medio natural y en una zona de pistas } \\
\text { para juegos tradicionales ( } 2 \text { horas). }\end{array}$} & $\begin{array}{l}\text { EFEPI } \\
\text { (CM1, } \\
\text { CM2) }\end{array}$ & $\begin{array}{l}\text { Juegos y actividades físico- } \\
\text { recreativas en el medio natural. } \\
\text { Juegos y actividades de } \\
\text { senderismo. } \\
\text { Juegos y actividades de } \\
\text { orientación. }\end{array}$ \\
\hline & $\begin{array}{l}\text { EFMNEP } \\
\text { (CA) }\end{array}$ & $\begin{array}{l}\text { Actividades para dinamizar } \\
\text { estancias en el medio natural. }\end{array}$ \\
\hline \multirow{2}{*}{ 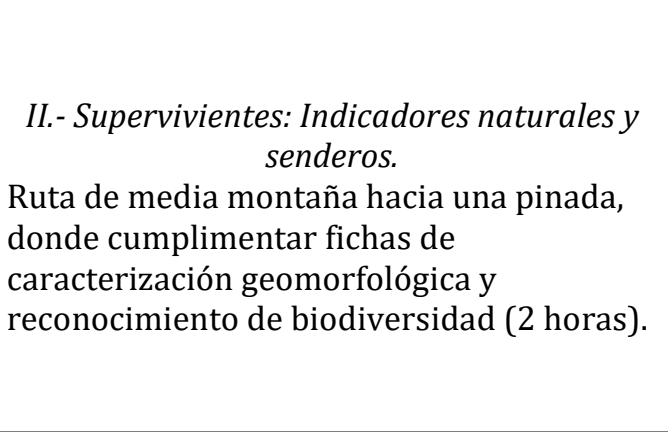 } & $\begin{array}{l}\text { DCCEE } \\
\text { (MD02, } \\
\text { MD06, } \\
\text { CM7, } \\
\text { CM8) }\end{array}$ & $\begin{array}{l}\text { Contenidos de ciencias en el } \\
\text { currículo oficial de Ed. } \\
\text { Primaria. } \\
\text { Actividades de enseñanza de } \\
\text { las ciencias en Ed. Primaria. }\end{array}$ \\
\hline & $\begin{array}{l}\text { EyAMNI } \\
\text { (MD01, } \\
\text { MD03, } \\
\text { MD04, } \\
\text { MD05) }\end{array}$ & $\begin{array}{l}\text { Los seres vivos y ecosistemas. } \\
\text { El medio y la sostenibilidad. } \\
\text { La materia y la energía: } \\
\text { diversidad, cambios y } \\
\text { propiedades. }\end{array}$ \\
\hline \multirow{5}{*}{$\begin{array}{l}\text { III.- Rastreator: Juego de orientación, rastreo } \\
\text { y pistas, identificando e interpretando un } \\
\qquad M T N \text {. } \\
\text { Localización de pistas: palabras que debían } \\
\text { ser ordenadas para responder en qué edad } \\
\text { geológica tuvo lugar la orogenia que } \\
\text { levantó topográficamente Sierra Espuña } \\
\text { (1.5 horas). }\end{array}$} & $\begin{array}{l}\text { CCSSD } \\
\text { (CM7) }\end{array}$ & $\begin{array}{l}\text { Desarrollo del currículo de } \\
\text { CC.SS. }\end{array}$ \\
\hline & \multirow{2}{*}{$\begin{array}{l}\text { ETDCCSS } \\
\text { (MD09) }\end{array}$} & $\begin{array}{l}\text { La dimensión espacial: } \\
\text { representación, orientación, } \\
\text { escala y simbología. }\end{array}$ \\
\hline & & $\begin{array}{l}\text { El espacio vivido: la relación } \\
\text { hombre-medio. }\end{array}$ \\
\hline & $\begin{array}{l}\text { MDECCSS } \\
\text { (CM5) }\end{array}$ & $\begin{array}{l}\text { Estrategias de enseñanza: } \\
\text { indagación mediante salidas y } \\
\text { trabajos de campo. }\end{array}$ \\
\hline & $\begin{array}{l}\text { ATCCSS } \\
\text { (CE2) }\end{array}$ & $\begin{array}{l}\text { Juegos de simulación: somos } \\
\text { geógrafos. }\end{array}$ \\
\hline $\begin{array}{l}\text { IV.- Exploradores: Organización de } \\
\text { actividades en el medio natural. } \\
\text { Organización ( } 2 \text { horas) y puesta en práctica } \\
\text { (4 horas) de actividades en el medio } \\
\text { natural dirigidas a estudiantes de } \\
\text { Educación Primaria, siguiendo una ficha } \\
\text { didáctica e inspirándose en lo aprendido en } \\
\text { los proyectos anteriores. }\end{array}$ & \multicolumn{2}{|c|}{$\begin{array}{c}\text { Aplicación práctica integral de los } \\
\text { contenidos de EF, CC.EE y CC.SS } \\
\text { (CB1, CB2, CB3, CGT1, CGT2, CGT3, CGT5, } \\
\text { CGT6, CGT7, CET1, CET2, CET4, CET5a, } \\
\text { CET6c, CET7a, CET9, CET10a, CET10b, } \\
\text { CET12a). }\end{array}$} \\
\hline $\begin{array}{l}\text { V.- La luna: Juegos grupales nocturnos. } \\
\text { Juegos nocturnos y dinámicas de grupo de } \\
\text { interacción con el medio natural ( } 2 \text { horas). }\end{array}$ & \multicolumn{2}{|r|}{$\begin{array}{l}\text { Dinámicas de grupo } \\
\text { (CGT3, CET5a). }\end{array}$} \\
\hline
\end{tabular}


*Nota. Códigos de las materias y cursos en que se imparten: DCCEE- Didáctica de las CC.EE ( $\left.2^{\circ}\right)$; EyAMNI Enseñanza y Aprendizaje del Medio Natural I ( $2^{\circ}$ ); CCSSD - CC.SS y su Didáctica (2ํㅜ); ETDCCSS - Espacio y Tiempo en la Didáctica de las CC.SS ( $\left.2^{\circ}\right)$; EyAMNII - Enseñanza y Aprendizaje del Medio Natural II ( $3^{\circ}$ ); EFEPI - EF en Educación Primaria I ( $3^{\circ}$ ); MDECCSS - Metodología didáctica para la enseñanza de las

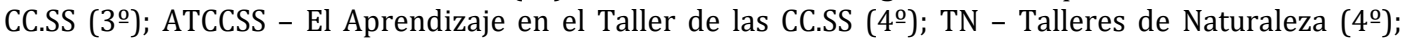
EFMNEP - EF en el Medio Natural en Educación Primaria (4ํㅜ).

Fuente y desarrollo de los contenidos y competencias ("Comp."): guías docentes de las materias correspondientes del Grado en Educación Primaria de la Universidad de Murcia ofertado por ISEN: https://www.um.es/web/isen/contenido/estudios/grados/edu-primaria/2018-19

\section{Instrumentos y métodos de evaluación}

Para cuantificar el grado de aprendizaje adquirido por el alumnado y su valoración sobre la eficacia didáctica y organizativa de la propuesta docente, se recurrió, en primer lugar, a un cuestionario de evaluación. Su diseño, basado en una evaluación competencial (Luna y Reyes, 2015), está estructurado en 4 bloques (tabla 2).

\section{Tabla 2}

Preguntas del cuestionario en relación con las competencias evaluadas y los bloques estructurales de la prueba

\begin{tabular}{lcc}
\hline Bloque & Código de pregunta & Código de competencia (materia)* \\
\hline 1) Identificación anonimizada & 0 & - \\
\hline & 1 & CM7 (CCSSD) \\
2) Aspectos formativos & 2 & MD09 (ETDCCSS) \\
(evaluación) & 3 & CM5 (MDECCSS) \\
Preguntas de respuesta breve & 4 & CE2 (ATCCSS) \\
sobre la didáctica de cada & 5 & MD01 (EyAMNI) \\
área (CC.SS: 1-4; CC.EE: 5-11; & 6 & MD03 (EyAMNI) \\
EF: 12-15). Se relacionan con & $7 \mathrm{a}$ & MD04 (EyAMNI) \\
las tareas abordadas y con los & $7 \mathrm{~b}$ & MD04 (EyAMNI) \\
contenidos curriculares y las & 8 & MD05 (EyAMNI) \\
competencias de las materias. & $9 \mathrm{a}$ & MD02, MD06 (DCCEE) \\
Se asume que haber & $9 \mathrm{~b}$ & MD02, MD06 (DCCEE) \\
alcanzado el conocimiento & 10 & CM7 (DCCEE) \\
máximo de los contenidos de & $11 \mathrm{a}$ & CM8 (DCCEE) \\
un área significa haber & $11 \mathrm{~b}$ & CM8 (DCCEE) \\
desarrollado al 100\% sus & $12 \mathrm{a}$ & CM1 (EFEPI) \\
competencias propias y, por & $12 \mathrm{~b}$ & CM1 (EFEPI) \\
tanto, haber adquirido el & 13 & CM2 (EFEPI) \\
grado máximo de aprendizaje & $14 \mathrm{a}$ & CA (EFMNEP) \\
posible. & $14 \mathrm{~b}$ & CA (EFMNEP) \\
& 15 & CGT1, CET1 \\
3) Aspectos formativos & & \\
ISSN0213-8646|E-ISSN2530-3791 $\bullet$ RevistaInteruniversitariadeFormacióndelProfesorado,96(35.3)(2021),225-246 & $\mathbf{2 3 1}$
\end{tabular}




\begin{tabular}{|c|c|c|}
\hline \multirow{7}{*}{$\begin{array}{l}\text { (percepción) } \\
\text { Preguntas de escala Likert } \\
\text { (rango de } 1 \text { a 4), más una de } \\
\text { respuesta abierta, sobre la } \\
\text { percepción de aprendizajes } \\
\text { adquiridos relacionados con } \\
\text { competencias básicas de la } \\
\text { etapa universitaria y } \\
\text { competencias generales y } \\
\text { transversales del Grado en } \\
\text { Educación Primaria. }\end{array}$} & 16 & $\begin{array}{c}\text { CGT1, CGT2, CGT3, CGT5, CGT6, } \\
\text { CGT7, CET2, CET4 }\end{array}$ \\
\hline & 17 & CGT5, CGT7, CET5a \\
\hline & 18 & $\begin{array}{c}\text { CGT1, CGT2, CGT6, CGT7, CET6c, } \\
\text { CET12a }\end{array}$ \\
\hline & 19 & $\begin{array}{c}\text { CGT1, CGT2, CGT3, CGT5, CET7a, } \\
\text { CET10b }\end{array}$ \\
\hline & 20 & CGT1, CGT2, CGT5, CGT7, CET9 \\
\hline & 21 & CGT1, CGT6, CET10a \\
\hline & 22 & CB1 \\
\hline & 23 & CB2 \\
\hline & 24 & CB3 \\
\hline $\begin{array}{l}\text { 4) Aspectos organizativos } \\
\text { Preguntas de escala Likert } \\
\text { (rango de } 1 \text { a } 4 \text { ) sobre la } \\
\text { valoración de la logística. }\end{array}$ & $25-38$ & - \\
\hline
\end{tabular}

*Nota. Ver Tabla1 para la codificación.

Este cuestionario de evaluación fue validado en un proceso de revisión de expertos, procedentes de los ámbitos de las Didácticas de las CC.SS, CC.EE y EF, que puntuaron en una escala Likert de 1 a 4 de menor a mayor adecuación y relevancia cada uno de los ítems de la prueba, aportando observaciones y sugerencias. El resultado fue muy positivo, con un nivel de adecuación medio total de 3.87 sobre 4 $(\mathrm{SD}=.19) \mathrm{y}$, de relevancia, de 3.99 sobre 4 (SD = .01). Los expertos sugirieron algunos matices en la redacción de ciertos ítems para lograr una mayor simplificación y comprensión de la prueba. Para asegurar la validez estadística de esta valoración conjunta inter-jueces por bloques se aplicó el coeficiente $W$ de Kendall, que mide el nivel de concordancia entre las respuestas de varios sujetos (Spooren et al., 2007), resultando en un valor moderado (.572, con valor $p<.05)$.

En segundo lugar, para extraer más información sobre la valoración del alumnado en aspectos formativos y organizativos, se recurrió al análisis del registro de una técnica de evaluación semicuantitativa adaptada de la conocida como "técnica del tendero de los deseos", llevada a cabo al final de la propuesta docente. Consiste en una evaluación formativa y compartida para recoger información más personalizada del proceso educativo (García-Herranz y López-Pastor, 2015) mediante la entrega al alumnado de una hoja en blanco en la que se le pide que escriba un "deseo", el cual exprese el momento, actividad, situación, etc., que más o menos le ha gustado a lo largo del Taller. Escrito el deseo, el alumnado coge una pinza y lo cuelga en el tendero. Cuando todos están tendidos, se dialoga sobre los mismos. Después, el alumnado debe poner una pinza en aquellos deseos de otros compañeros con los que esté de acuerdo, conociéndose y explicitándose así la autoevaluación efectuada por los participantes. 
Esta técnica posibilitó una evaluación abierta, registrada en una hoja de cálculo para cuantificar tipos de impresiones y su nivel de acuerdo por parte del alumnado. Tiene un uso mayoritario en la educación infantil, pero se puede utilizar en otras etapas desarrollando adaptaciones psicopedagógicas (López-Pastor y Pérez-Pueyo, 2017).

\section{Análisis estadísticos}

Por un lado, se establecieron valores de 1 a 4, en función de menor o mayor corrección, para las respuestas del bloque 2 del cuestionario de evaluación, entendiendo estos valores como el rango de competencia adquirido. Por otro lado, se calculó el valor relativo de competencia de cada estudiante, sumando los valores de todas sus respuestas, así como el valor relativo de competencia promediado entre alumnos. Finalmente, estos valores se normalizaron, en datos porcentuales, como la proporción de logro alcanzado por cada alumno en cada área, así como la proporción de logro alcanzado en promedio entre todos los alumnos. Quedaron definidas así las variables que describen la competencia o conocimiento medio mostrado en cada área.

Paralelamente, se establecieron tres categorías de respuesta para los valores de desarrollo competencial: "bien" (3-4), "mal" (1-2) y "no saben/no contestan" (en blanco). Para cada ítem y también de forma promediada entre ítems, se calculó la proporción de alumnos, en porcentaje, en cada categoría. Este mismo tratamiento se realizó para las variables "AF" (aspectos formativos y didácticos) y "AO" (aspectos organizativos), derivadas de los bloques 3 y 4 del cuestionario.

En cuanto a los resultados del "tendero de los deseos", que complementan los anteriores, se realizó una reclasificación, agrupando en 4 categorías los comentarios registrados: aspectos formativos (AF), aspectos organizativos (AO), aspectos psicológicos motivacionales (APM), y aspectos de relación social (ARS).

Los datos fueron sometidos a análisis estadísticos mediante el programa SPSS, Versión 2017 para Windows. Se analizó la relación entre dos grupos de variables: 1) las variables descriptoras de la competencia en cada área específica calculadas a partir del bloque 2 del cuestionario (CO_código del área) y 2) variables que representaban las calificaciones obtenidas por cada alumno en cada área, extraídas de sus expedientes académicos del Grado (CA_código del área). En el caso de las áreas de CC.SS y CC.EE, al haber cursado los alumnos tres asignaturas en cada una, se calculó el promedio entre las calificaciones.

Tras comprobar la normalidad de las variables, se realizaron análisis de la varianza (ANOVA de un factor) y análisis de correlación de Pearson, para comprobar la posible existencia de diferencias significativas en la distribución de los datos y detectar posibles correlaciones, dentro de cada área, entre la competencia mostrada en el cuestionario y la calificación obtenida en la(s) correspondiente(s) asignatura(s) del Grado. 


\section{Resultados}

\section{Grado de aprendizaje}

Como se aprecia en la tabla 3, CC.SS es, de las tres áreas específicas, aquella en la que los alumnos muestran un mayor logro (77.6\%), es decir, una mejor aplicación de los conocimientos adquiridos durante el Grado, con un valor medio de respuesta de 3.36 sobre 4 y un valor total medio por alumno de 12.4 sobre 16 . Por su parte, el área de EF a es la que muestra un menor logro o competencia (41.2\%), con un valor de respuesta de 2.51 sobre 4 y un valor total medio por alumno de 8.2 sobre 20 . En una situación intermedia se sitúa el área de CC.EE, con un $52.8 \%$ de logro y un valor medio de respuesta de 2.64 sobre 4 y de 21.1 sobre 40 como valor total medio por alumno.

\section{Tabla 3}

Grado de competencia y porcentaje de logro alcanzado por área (valores sumados de los ítems y promediado entre alumnos)

\begin{tabular}{lccc}
\hline Competencia_Área & CO_CS & CO_CE & CO_EF \\
\hline Valor medio & 3.36 & 2.64 & 2.51 \\
SD & .66 & .68 & .87 \\
Mediana & 3.50 & 2.70 & 2.30 \\
№ Preguntas & 4 & 10 & 5 \\
Valor máximo total & 16 & 40 & 20 \\
Valor total promedio (alumnos) & 12.4 & 21.1 & 8.2 \\
Logro alcanzado (en \% y promedio entre alumnos) & 77.6 & 52.8 & 41.2 \\
\hline
\end{tabular}

El grado de aprendizaje según categorías de respuesta se puede observar en la figura 2. En el área de CC.SS, la mayoría de respuestas son total o mayoritariamente correctas $(84.5 \%)$ frente a un $7.76 \%$ de respuestas total o parcialmente incorrectas. Contrasta de nuevo con el área de EF, en la que existe prácticamente la misma proporción de respuestas incorrectas (31.03\%) que correctas (31.72\%). Destaca, tanto en CC.EE como en EF, una importante proporción de respuestas en blanco (21.03\% y $37.24 \%$ respectivamente). 


\section{Figura 2}

Proporción (\%) de las categorías de respuestas en cada área

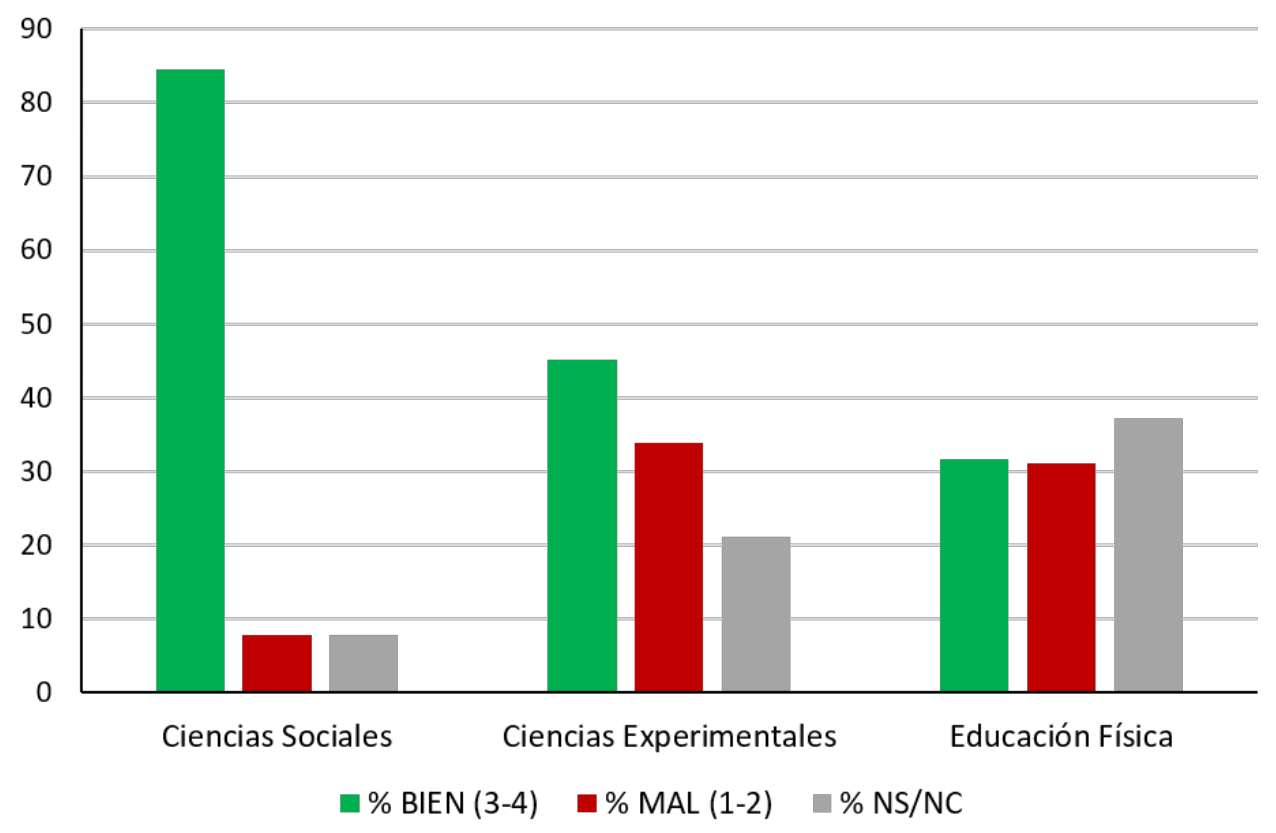

En la figura 3, se muestra de manera gráfica la proporción de las diferentes categorías de respuestas para cada pregunta del cuestionario y para cada área específica. En CC.SS, existe un predominio claro de respuestas correctas, que solo baja del $80 \%$ en la P4, aunque ligeramente. En el área de CC.EE, destaca cómo en las preguntas relacionadas con aspectos de didáctica de las ciencias (P9 a P11) se reduce notablemente la proporción de respuestas correctas (siendo menos de un $20 \%$ en P9b, P11a y P11b) y aumentan las respuestas incorrectas o en blanco. Esto evidencia, en el área de CC.EE, un mejor dominio de aspectos teóricos y conceptuales (P5 a P8) que didácticos y pedagógicos (P9 a P11). En EF, aparecen resultados con una gran dispersión, incluyendo desde preguntas con un porcentaje de acierto inexistente (P12a) hasta preguntas con un $0 \%$ de fallo (P14a). Destaca en esta área una proporción recurrente de respuestas en blanco en todos los ítems (desde un $13.8 \%$ en P13 hasta un $72.4 \%$ en P14b). 


\section{Figura 3}

Proporción (\%) de las categorías de respuestas en CC.SS, CC.EE y EF

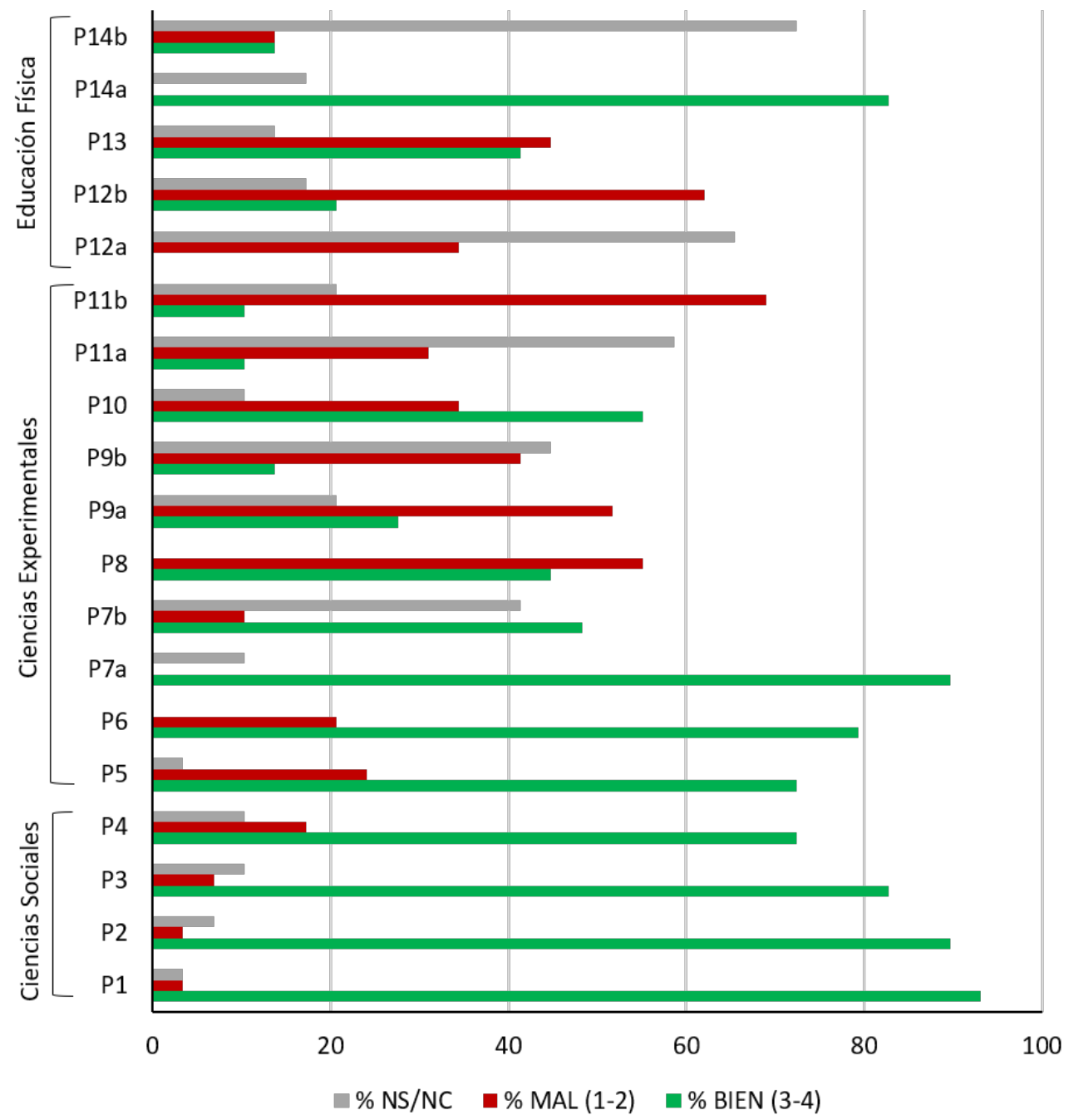

Realizando en los datos anteriores análisis de varianza y de correlación (tabla 4), lo más destacable es la ausencia de diferencias significativas entre el conocimiento mostrado en el taller y la calificación media obtenida durante el Grado en las áreas de CC.SS y CC.EE. En el caso de CC.SS, además, aparece una correlación positiva y significativa entre el CO y la CA: el conocimiento adquirido en el grado es aplicado y demostrado en el taller a través de las respuestas dadas por los alumnos. La ausencia de correlación entre CO_CE y CA_CE, pese al resultado del ANOVA, podría estar explicada por la mayor dispersión de los datos en CO_CE. La calificación media en ambas áreas es bastante cercana (7.3 en CC.EE y 7.67 en CC.SS), aunque el valor de 
competencia medio en la primera (2.67) es bastante más bajo que en la segunda (3.34). A esto se le une que los rangos en los que se mueve la desviación estándar y la mediana en CC.SS son mucho menores (DS = .6 - .7 y $\mathrm{M}=3-4$, según el ítem) que en CC.EE (DS = .4 - 1 y M = 2 - 4, según el ítem). En el área de EF, por su parte, sí se observan diferencias significativas entre CO y CA, encontrándose una correlación positiva y significativa. Las diferencias detectadas pueden ser debidas a que CO_EF es la que tiene un valor medio y un porcentaje de logro alcanzado más bajo de las tres áreas específicas, sin embargo, CA_EF presenta un valor medio muy cercano a las otras dos áreas. La correlación positiva puede ser explicada debido a que los alumnos con menor CA_EF son los que presentan una menor competencia en EF en el taller, aunque las medias de las distribuciones muestren diferencias significativas.

\section{Tabla 4}

Resultados ANOVA y Correlaciones de Pearson

\begin{tabular}{lccc}
\hline Variables & Sig. ANOVA & Coef. Cor. Pearson & Sig. Cor. Pearson \\
\hline CO_CS vs CA_CS & .127 & .39 & $.037^{*}$ \\
CO_CE vs CA_CE & .815 & .11 & .568 \\
CO_EF vs CA_EF & $.033^{*}$ & .478 & $.01^{*}$ \\
\hline
\end{tabular}

\section{Valoración didáctica y logística}

La valoración de los aspectos didáctico-formativos (AF) y logísticos y organizativos (AO) se puede apreciar en la tabla 5, considerando los valores promediados entre ítems y alumnos. El grado de valoración por parte de los participantes en ambos aspectos fue elevado, con casi un 95\% de respuestas satisfactorias (valores 3 y 4) y casi un $87 \%$ de valoración global. Se puede afirmar que los alumnos comprenden y valoran la intencionalidad formativa del taller y entienden la importancia y posibilidades didácticas de este tipo de enfoques integrales y activos (preguntas 15 a 24) y, al mismo tiempo, valoran positivamente su organización, adecuación, secuenciación y temporalización (preguntas 25 a 38).

\section{Tabla 5}

Grado de valoración de los aspectos formativos (AF) y aspectos organizativos (AO)

\begin{tabular}{lcc}
\hline & AF & AO \\
\hline Media (entre ítems) & 3.54 & 3.55 \\
Desviación & .51 & .54 \\
Mediana & 3.70 & 3.54 \\
\% BIEN (3-4) & 94.83 & 94.83 \\
\% MAL (1-2) & 3.10 & 2.96
\end{tabular}


$\%$ NS/NC

№ Preguntas

Valor máximo total

Valoración total promedio (alumnos)

Valoración total (en \% y promedio entre alumnos)
2.07

10

40

34.79

86.98
2.22

14

56

48.59

86.76

El análisis del registro del "tendero de los deseos" permitió complementar la valoración y evaluación realizada por el alumnado. Esta evaluación abierta mostró otros aspectos como elementos clave en el proceso educativo, de tal forma que el alumnado relacionó un mayor grado de satisfacción con aspectos psicológicos motivacionales (APM), con un total de 27 pinzas (36.5\%). Los aspectos de relación social (ARS) fueron considerados el segundo tipo con alto grado de impresión en la satisfacción, recibiendo un total de 20 pinzas (27\%). Los AO, con 18 pinzas (24.3\%), y los $\mathrm{AF}$, con 9 (12.2\%), fueron los menos comentados y valorados, pero igualmente recibieron una cierta atención.

\section{Discusión}

La salida didáctica al espacio natural de Sierra Espuña ha propiciado el desarrollo de competencias relativas a la formación de maestros, en línea con multitud de experiencias similares (Costillo et al., 2014; Delgado Huertos, 2015; Rebar y Enochs, 2010), si bien es cierto que aquí las competencias relacionadas con el área de CC.SS se han manifestado de forma más evidente que las de las áreas de CC.EE y EF.

Las competencias buscadas desde el enfoque de las CC.SS están fundamentalmente relacionadas con el pensamiento espacial y la capacidad de orientación, así como su tratamiento didáctico. Se trata de competencias de desarrollo cotidiano que tienen un carácter más procedimental que conceptual (Uttal et al., 2013), por lo que su puesta en práctica puede generar un aprendizaje más significativo, con resultados más directos que los propiciados con otro tipo de competencias más vinculadas a aspectos teóricos.

La relación de los contenidos con el medio es una idea muy trabajada teóricamente en la didáctica de las CC.SS (Vilarrasa Cunillé, 2005), sobre todo desde la Geografía, concebida como la relación entre la actividad humana y el medio natural (Parra Monserrat y Morote Seguido, 2020), y esto permitido un traslado directo del aprendizaje dentro del aula a fuera de esta. Sin embargo, en CC.EE, con contenidos en este caso más vinculados a conceptos naturales que a procedimientos de experimentación, no se ha producido ese traslado de forma predominante, de modo que los estudiantes han sido mayoritariamente incapaces de relacionar de forma correcta la teoría de clase con su materialización en el medio natural. Precisamente uno de los objetivos más perseguidos por las salidas didácticas es propiciar esa relación entre la abstracción de los contenidos teóricos y su concreción en una realidad material (Domínguez Almansa y López Facal, 2014). En la literatura científica se habla de "educación ambiental" para lograr esta relación (Dale et al., 2020) y que, como consecuencia actitudinal, se alcance una concienciación 
ambientalista (Stern et al., 2008), considerando a los estudiantes como agentes de cambio (Fielding, 2001). Se deriva de nuestros resultados que una única salida didáctica puede no ser suficiente para lograr de forma completa esta educación ambiental, sí de forma inicial, así que parece aconsejable, como proponen Morag et al. (2013), plantear planificaciones didácticas a más largo plazo apoyadas en salidas sucesivas fuera del aula.

Probablemente, en el área de EF también se ha dado un problema de conexión entre la teoría de clase, muy vinculada al espacio cerrado del pabellón y del patio escolar, y su puesta en práctica fuera del aula, en un espacio abierto y con escasos elementos antrópicos. Esta carencia de capacidad de vinculación ha resultado en un desarrollo incompleto de las competencias propias del área, muy relacionadas con un aprendizaje en sintonía con el medio natural y, por tanto, de carácter interdisciplinar. Se trata de un problema reconocido en la didáctica de la EF, ya que la naturaleza es un espacio poco habitual para la práctica escolar de la materia (Santos-Pastor y Martínez Muñoz, 2011), y precisamente la potenciación de salidas didácticas al medio se postula como una de las posibles soluciones, desde la base de la interdisciplinaridad (Gallego-Lema et al., 2019).

No obstante, aunque los estudiantes no han alcanzado todo el desarrollo competencial previsto, sí han sido conscientes de que una práctica docente fuera del aula es una gran ayuda para conseguirlo, y han valorado muy positivamente la formación general que propician las salidas didácticas como la que llevaron a cabo, a través de un aprendizaje interdisciplinar con base en el medio natural. De esta forma, el medio actúa de argamasa para una educación integral, lo que constituye una de las fortalezas de las salidas didácticas (Crespo et al., 2018). Esta idea ha sido muy abordada. Benejam (2003) se refiere a las salidas como "experiencias integrales". DeWitt y Storksdieck (2008) estudian cómo las salidas tienen más utilidad como experiencias de descubrimiento integral que como método de enseñanza de conceptos puntuales. Nazareth et al. (2019) incluso vinculan la adquisición de competencias de situación y orientación en el medio con el mejor desarrollo de las disciplinas STEM (Ciencia, Tecnología, Ingeniería y Matemáticas). Debido a estas consideraciones, la salida a Sierra Espuña no solo encuentra su eficacia didáctica en el desarrollo de competencias propias de determinadas áreas de conocimiento sino también en su concepción interdisciplinar.

El aprendizaje por competencias es clave en las experiencias de innovación educativa (Cañadas et al., 2020). Además de las competencias propias de cada área y de aquellas trasversales y básicas que han motivado esta práctica docente, la salida a Sierra Espuña también ha permitido que los estudiantes desarrollasen algunas de las competencias que, según la Orden ECI/3857/2007, forman parte de los requisitos para la validación de un estudio superior de magisterio: 1) Conocer las áreas curriculares de la Educación Primaria, la relación interdisciplinar entre ellas (...); 2) Diseñar, planificar y evaluar procesos de enseñanza y aprendizaje, tanto individualmente como en colaboración con otros docentes y profesionales del centro; 5) Fomentar la convivencia en el aula y fuera de ella, resolver problemas de disciplina y contribuir a la resolución pacífica de conflictos (...); y 9) Valorar la responsabilidad individual y colectiva en la consecución de un futuro sostenible. 
Todas las competencias también se desarrollan dentro del aula, pero el tratamiento completo de muchas de ellas depende de actividades fuera del centro educativo. No se han observado diferencias significativas entre las calificaciones de clase de las áreas de CC.SS y CC.EE y el logro de competencia alcanzado en la salida para cada área, lo que pone de manifiesto la relación que existe entre el aprendizaje que se produce fuera del aula y el que se produce dentro. Solo en el área de EF ha habido diferencias significativas, probablemente derivadas de la mencionada carencia de vinculación entre la EF impartida de forma convencional y aquella que se apoya en un espacio natural abierto, que, tal como se deduce del amplio porcentaje de abstenciones en las preguntas de evaluación, ha generado una gran confusión entre el alumnado. Esta confusión también se ha dado en CC.EE, pero más reflejada en errores académicos, proporcionales al nivel de calificación de cada estudiante, que en el error estructural de concepción que se ha producido en la didáctica de la EF llevada a la naturaleza, transversal en todo el alumnado. Quizá en estas áreas haya que prestar mayor atención a la vinculación de los contenidos con el medio natural antes de salir del aula.

Por último, desde un punto de vista organizativo y metadidáctico, los estudiantes han mostrado una satisfacción muy elevada con la práctica docente. Han sido muchos los que han puesto en valor aspectos psicológicos motivacionales, con afirmaciones como: "todas las actividades han sido muy adecuadas y divertidas, ha sido mucho mejor de lo que esperábamos" o "me ha dado muchas ideas para el futuro". También han destacado los aspectos de relación social; por ejemplo, los estudiantes reconocen que de esta propuesta les "ha gustado la integración con los compañeros" o que "nos hemos conocido mejor, olvidando las diferencias presentes en el aula".

Resulta llamativo que los estudiantes destaquen aspectos emocionales sobre los didácticos. Otros autores como Heras et al. (2020), también constataron que las salidas de campo producen antes un beneficio emocional y social que cognitivo. Rajala y Akkerman (2019), por su parte, igualmente ponen el foco en la importancia de las emociones de los participantes en salidas didácticas, proponiendo un aprendizaje dialógico. No obstante, este beneficio emocional y social constituye asimismo un aprendizaje, focalizado en el desarrollo personal (Council for Learning Outside the Classroom, 2006), y probablemente suponga una condición muy favorable para facilitar paralelamente un aprendizaje ambiental y, también, un aprendizaje de corte más académico (Dale et al., 2020; Farmer et al., 2007; Stern et al., 2008), apoyado en un aumento en la motivación del alumnado y en sus habilidades de indagación (Del Toro y Morcillo, 2011).

Los resultados de aprendizaje competencial obtenidos en esta investigación avalan estas consideraciones, de forma más evidente en el área de CC.SS. Se puede afirmar que, en esta experiencia proporcionada a los futuros docentes fuera del contexto del aula, lo que según la literatura científica no es muy habitual a pesar de los múltiples estudios sobre sus beneficios, ha habido un aprendizaje vivencial elevado que ha facilitado un aprendizaje trasversal también considerable pero un aprendizaje específico variable según el área de conocimiento. 


\section{Conclusiones}

Los maestros en formación desarrollan competencias que los habilitan para el ejercicio de la profesión docente. Sin embargo, algunas pueden adquirir una dimensión más completa si se trabajan también fuera del aula, mediante salidas didácticas, que, a partir de un espacio geográfico, pongan en relación las competencias que en clase se presentan de forma estanca a través de materias diferentes.

Ya hay multitud de estudios sobre experiencias de salidas. La que se presenta en esta investigación tiene como peculiaridad que ha permitido de forma explícita la interdisciplinaridad entre las áreas de CC.SS, CC.EE y EF, a través de la implementación de una práctica docente estructurada en proyectos y desarrollados de forma secuencial hasta un tratamiento interdisciplinar.

La eficacia didáctica de la salida ha sido notable, por el grado de desarrollo de las competencias propias de la formación de maestros (más evidente en CC.SS, probablemente por una asociación automática y casi insoslayable de los contenidos con el medio natural). Asimismo, los estudiantes han sabido percibir su importancia para hacer implícitos, significativos y relacionables los aprendizajes del aula, así como para adquirir una experiencia de indagación desde el compañerismo y la motivación.

Estos resultados refuerzan la idea de que las salidas didácticas propician un aprendizaje interdisciplinar y fortalecen la adquisición de competencias que dentro del aula pueden quedar incompletas, como las relativas al trabajo con otros compañeros, la concienciación ambiental o la capacidad de vincular conocimientos abstractos e independientes a una realidad tangible e interrelacionada. Por todo ello, parece razonable reclamar que las salidas tengan una mayor presencia curricular. Esto además tendrá repercusión en la trasposición con los futuros estudiantes de educación primaria, a los que se inculcará la importancia de salir del aula $\mathrm{y}$, consecuentemente, un conocimiento más holístico del medio y una mayor sensibilización ambiental.

No obstante, a raíz de las diferencias de logro, no parece recomendable conformarse con una única experiencia para ser capaz de relacionar de forma completa los contenidos y las competencias trabajados en clase con los requeridos fuera, sobre todo en disciplinas donde la vinculación con el medio es más abstracta desde la impartición teórica.

Finalmente, se deben tener presentes algunas limitaciones de nuestro estudio, que hacen que los resultados deban considerarse prudentemente. Se trata de la muestra utilizada, de reducido tamaño, y de la puntualidad de la experiencia. También hay que tener en cuenta el inevitable halo de subjetividad que puede escapar en la valoración de los instrumentos de evaluación.

\section{Agradecimientos}

Esta investigación se llevó a cabo en el marco del Proyecto del Ministerio de Ciencia, Innovación y Universidades del Gobierno de España "Conceptos metodológicos y métodos activos de aprendizaje para la mejora de las competencias docentes de profesorado" (Referencia PGC2018-094491-B-C33). 


\section{Conflicto de intereses}

Los autores declaran no tener ningún conflicto de intereses. La recopilación de datos para la investigación se realizó siguiendo las Orientaciones Éticas para la Investigación Social Comparativa Internacional del código ético de investigación en Ciencias Sociales de la UNESCO, destacando el anonimato, confidencialidad, información continua a los participantes y la justificación de los métodos aplicados.

\section{Contribuciones de los autores}

Conceptualización y planteamiento teórico, C.M-H., MI.C-I. y FJ.R-M.; metodología y análisis, C.M-H y P.F-C.; discusión científica: C.M-H. y MI.C-I.; redacción del borrador original, C.M-H.; redacción, revisión, edición y supervisión, C.M-H., MI.C-I., FJ.R-M. y P.F-C.

\section{Referencias}

Aguilera, D. (2018). La salida de campo como recurso didáctico para enseñar ciencias. Una revisión sistemática. Revista Eureka sobre Enseñanza y Divulgación de las Ciencias, 15(3), 3103-3119. https:// doi.org/10.25267/Rev_Eureka_ensen_divulg_cienc.2018.v15.i3.3103

Albaladejo, J. \& López, D. (2020). Salidas escolares para el conocimiento del medio natural, social, cultural y artístico en educación infantil: Estudio de caso. En Actas del IV Congreso Internacional de Investigación e Innovación en Educación Infantil y Primaria (pp. 73-76). Editum. http://hdl.handle.net/10201/87076

Amor, M.I. \& Serrano-Rodríguez, R. (2019). The generic competences in the initial teacher training. A comparative study among students, teachers and graduates of university education degree. Educación XX1, 22(1), 239-261. https://doi.org/10.5944/educXX1.21341

Arroyo, M.J., Pinedo, R. \& De la Iglesia, M. (2020). Coordinación docente e interdisciplinariedad para la adquisición de competencias en el Grado de Educación Primaria e Infantil: Percepciones de alumnado y profesorado. Tendencias Pedagógicas, 35, 102-117. https://doi.org/10.15366/tp2020.35.009

Asún, R., Zúñiga, C. \& Ayala, M.C. (2013). La formación por competencias y los estudiantes: confluencias y divergencias en la construcción del docente ideal. Revista Calidad en la Educación, 38, 277-304. https://doi.org/10.4067/S071845652013000100008

Benejam, P. (2003). Los objetivos de las salidas. Iber: Didáctica de las ciencias sociales, geografía e historia, 36, 7-12.

Cano, M.E., Pons, L. \& Lluch, L. (2018). Análisis de experiencias de innovación docente universitarias sobre evaluación. Profesorado. Revista de Currículum y Formación de profesorado.v22i4.8392

Profesorado, 22(4), 11-32. https://doi.org/10.30827/

Cañadas, L., Santos-Pastor, M.L. \& Castejón, F.J. (2020). Physical Education Teachers' Competencies and Assessment in Professional Practice. Apunts. Educación Física y Deportes, 139, 33-41. https://doi.org/10.5672/apunts.20140983.es.(2020/1).139.05 
Costillo, E., Borrachero, A.B., Esteban, M.R. \& Sánchez-Martín, J. (2014). Aportaciones de las salidas al medio natural como actividades de enseñanza y de aprendizaje según profesores en formación. Indagatio didáctica, 6(3) 10-22. http://dx.doi.org/10.34624/id.v6i3.3983

Council for Learning Outside the Classroom (2006). Learning Outside the Classroom. Manifesto. DfES Publications.

Crespo, J.M., Gómez, M.L. \& Cruz, L.A. (2018). Una aproximación a los Parques Nacionales y sus paisajes a través de itinerarios didácticos. Espacio, Tiempo y Forma, 11, 121-140. https://doi.org/10.5944/etfvi.11.2018.22359

Dale, R., Powell, R., Stern, M. \& Garst, B. (2020). Influence of the natural setting on environmental education outcomes. Environmental Education Research,

26(5), 613-631. https://doi.org/10.1080/13504622.2020.1738346

Decreto 13/1995, de 31 de marzo, por el que se aprueba el PORN de Sierra Espuña (incluido Barrancos de Gebas) y se declara como paisaje protegido los Barrancos de Gebas. BORM 85, del 11 de abril de 1995.

Del Toro, R. \& Morcillo, J.G. (2011). Las actividades de campo en Educación Secundaria. Un estudio comparativo entre Dinamarca y España. Enseñanza de las Ciencias de la Tierra, 19(1), 39-47. https:// www.raco.cat/index.php/ECT/article/view/244377/331349

Delgado Huertos, E. (2015). The landscape in the training of teachers, an educative resource of great interest in primary education. TABANQUE Revista pedagógica, 28, 117-138. https://core.ac.uk/download/pdf/211099265.pdf

DeWitt, J. \& Storksdieck, M. (2008). A short review of school field trips: Key findings from the past and implications for the future. Visitor Studies, 11(2), 181-197. https://doi.org/10.1080/10645570802355562

Domínguez Almansa, A. \& López Facal, R.V. (2014). Patrimonio, paisaje y educación: formación inicial del profesorado y educación cívica del alumnado de primaria. CLIO. History and History teaching, 40, 1-26. http://clio.rediris.es/n40/articulos/mono/MonDominguezLopez2014.pdf

Farmer, J., Knapp, D \& Benton, G. (2007). An Elementary School Environmental Education Field Trip: Long-Term Effects on Ecological and Environmental Knowledge and Attitude Development. The Journal of Environmental Education, 38(3), 3, 33-42. https://doi.org/10.3200/JOEE.38.3.33-42

Fielding, M. (2001). Students as Radical Agents of Change. Journal of Educational Change, 2(2), 123-141. https://doi.org/10.1023/A:1017949213447

Freire, P. (1990). La naturaleza política de la Educación: Cultura, Poder y Liberación. Paidos-MEC.

Gabarda, V. (2015). Buenas prácticas en educación. Universidad de Valencia.

Gallego-Lema, V., Muñoz-Cristóbal, J.A., Arribas Cubero, H. \& Rubia-Avi, B. (2019). El prácticum en educación física en el medio natural: Conectando espacio físicos y virtuales. Movimiento: revista da Escola de Educação Física, 1-16. https://doi.org/10.22456/1982-8918.81980

García-Herranz, S. \& López-Pastor, V.M. (2015). Evaluación Formativa y Compartida en Educación Infantil. Revisión de una Experiencia Didáctica. Qualitative Research in Education, 4(3), 269-298. https://doi.org/10.17583/qre.2015.1269 
Heras, R., Medir, R.M. y Salazar, O. (2020). Children's perceptions on the benefits of school nature field trips. Education 3-13,48(4), 379-391. https://doi.org/10.1080/03004279.2019.1610024

Lavega, P., Sáez de Orcáriz, U., Lasierra, G., \& Salas, C. (2013). Intradisciplinariedad e Interdisciplinariedad en la adquisición de competencias: estudio de una experiencia de aprendizaje cooperativo. Revista Electrónica Interuniversitaria de Formación del Profesorado, 16(1), 133-145. https://doi.org/10.6018/reifop.16.1.179491

López Facal, R.V. (2013). Competencias y enseñanza de las ciencias sociales. Íber: Didáctica de las ciencias sociales, geografía e historia, 74, 5-8.

López-Pastor, V.M. (2011). El papel de la evaluación formativa en la evaluación por competencias: aportaciones de la red de evaluación formativa y compartida en docencia universitaria. Revista de Docencia Universitaria, 9(1), 159-173. https://doi.org/10.4995/redu.2011.6185

López-Pastor, V.M. \& Pérez-Pueyo, A. (2017). Evaluación formativa y compartida en educación: experiencias de éxito en todas las etapas educativas. Servicio de Publicaciones Universidad de León.

Luna, E. (2000). Aspectos implícitos sobre la enseñanza reflejados en los cuestionarios de evaluación de la docencia. En M. Rueda y F. Díaz-Barriga (Eds.), Evaluación de la docencia. Perspectivas actuales (pp. 63-83). Paidós.

Luna, E. \& Reyes, E.P. (2015). Validación de constructo de un cuestionario de evaluación de la competencia docente. Revista Electrónica de Investigación Educativa, 17(3), 13-27. http://redie.uabc.mx/vol17no3/contenido-lunapinuelas.html

Maller, C. (2009). Promoting children's mental, emotional and social health through contact with nature: a model. Health Education, 109 (6), 522-543. https://doi.org/10.1108/09654280911001185

Martínez, M.J., Sánchez, M.C. \& García, F.A. (2012). Recursos para la innovación de la enseñanza y el aprendizaje en Educación Superior en España: Portafolios y Web-did ctica. Revista Iberoamericana de Educación, 59(1), 1-10. https:// doi.org/10.35362/rie5911400

Martínez-Hernández, C. y Yubero, C. (2020). Explaining Urban Sustainability to Teachers in Training through a Geographical Analysis of Tourism Gentrification in Europe. Sustainability, 12(67), 1-19. https://doi.org/10.3390/su12010067

Martínez-Hernández, C., Yubero, C., y Robles Moral, F.J. (2021). Itinerario didáctico multidisciplinar en Madrid: validación con maestros en formación. REDIE, Revista Electrónica de Investigación Educativa, 23, en prensa.

Mogensen, F. \& Schnack, K. (2010). The action competence approach and the 'new' discourses of education for sustainable development, competence and quality criteria. Environmental Education Research, 16(1), 59-74. https:// doi.org/10.1080/13504620903504032

Morag, 0., Tal, T. \& Rotem-Keren, T. (2013). Long-term educational programs in nature parks: Characteristics, outcomes and challenges. International Journal of Environmental and Science Education, 8(3), 427-449. https:// doi.org/ https://doi.org/10.12973/ijese.2013.213a 
Moreno-Vera, J.R. \& Monteagudo Fernández, J. (2019). Aprender historia del arte mediante salidas didácticas. Una experiencia en educación superior. Historia y Espacio, 15(53), 249-270. https://doi.org/10.25100/hye.v15i53.8660

Nazareth, A., Newcombe, N., Shipley, T., Velazquez, M. \& Weisberg, S. (2019). Beyond small-scale spatial skills: Navigation skills and geoscience education: Principles and implications principles and implications. Cognitive Research, 4(1), 1-17. https://doi.org/10.1186/s41235-019-0167-2

Orden ECI/3857/2007, de 27 de diciembre, por la que se establecen los requisitos para la verificación de los títulos universitarios oficiales que habiliten para el ejercicio de la profesión de Maestro en Educación Primaria. BORM 312, del 29 de diciembre de 2007.

Parra Montserrat, D. \& Morote Seguido, A.F. (2020). Memoria escolar y conocimientos didáctico-disciplinares en la representación de la educación geográfica e histórica del profesorado en formación. RIFOP. Revista Interuniversitaria de Formación del Profesorado, 34(3), 11-32. https://doi.org/10.47553/rifop.v34i3.82028

Peasland, E., Henri, D., Morrell, L. \& Scott, G. (2019). The influence of fieldwork design on student perceptions of skills development during field courses. International Journal of Science Education, 41(17), 2369-2388. https:// doi.org/10.1080/09500693.2019.1679906

Pedrinaci, E. (2012). Trabajo de campo y aprendizaje de las ciencias. Alambique: Didáctica de las Ciencias Experimentales, 71, 81-89.

Prats Cuevas, J. (2001). Valorar el patrimonio histórico desde la educación: factores para una mejor utilización de los bienes patrimoniales. Aspectos didácticos de las ciencias sociales, $15,157-171$.

Rajala, A. \& Akkerman, S. (2019). Researching reinterpretations of educational activity in dialogic interactions during a fieldtrip. Learning, Culture and Social Interaction, 20, 32-44. https://doi.org/10.1016/j.lcsi.2017.10.006

Rebar, B. \& Enochs, L. (2010). Integrating Environmental Education Field Trip Pedagogy into Science Teacher Preparation. En A. Bodzin, B. Shiner Klein y S. Weaver (Eds.), The Inclusion of Environmental Education in Science Teacher Education (pp. 111-126), Springer. https://doi.org/10.1007/978-90-481-9222$\underline{9 \_8}$

Rebelo, D., Marques, L. \& Costa, N. (2011). Actividades en ambientes exteriores al aula en la Educación en Ciencias: contribuciones para su operatividad. Enseñanza de las Ciencias de la Tierra, 19(1), 15-25. https:// www.raco.cat/index.php/ECT/article/view/244375

Redecker, C. \& Punie, Y. (2013). The future of learning 2025: developing a vision for change. Future Learning, 3-17. https://publications.jrc.ec.europa.eu/repository/handle/JRC85225 Rodríguez,

J.A. \& Santana, P.J. (2015). La distancia entre el trabajo del profesorado y del alumnado en el EEES: ¿una brecha peligrosa? Profesorado. Revista de Currículum y Formación de Profesorado, 19(3), 380-404. http://hdl.handle.net/10481/39918 
Santos-Pastor, M.L. \& Martínez Muñoz, F. (2011). Las actividades en el medio natural en la Educación Física de la escuela rural. Un estudio de caso. Profesorado, Revista de currículum y formación del profesorado, 15(2), 219-233. http://hdl.handle.net/10481/17442

Solbes, J. (2011). ¿Por qué disminuye el alumnado de ciencias? Alambique: Didáctica de las Ciencias Experimentales, 67, 53-61. https://www.uv.es/jsolbes/documentos/Alambique_Solbes_2011.PDF

Sousa Fernandes, S.A., García Monteagudo, D. \& Souto González, X.M. (2016). Educación geográfica y las salidas de campo como estrategia didáctica: un estudio comparativo desde el Geoforo Iberoamericano. Biblio3W, Revista Bibliográfica de Geografía y Ciencias Sociales, 21(1155). http://www.ub.edu/geocrit/b3w-1155.pdf

Souto González, X.M. (2011). Una educación geográfica para el Siglo XXI: Aprender competencias para ser ciudadano en el mundo global. Revista Anekumene, Revista Virtual de Geografía, Cultura y Educación, 1, 28-47. https://doi.org/10.17227/Anekumene.2011.num1.7079

Spooren, P., Mortelmans, D. \& Denekens, J. (2007). Student evaluation of teaching quality in higher education: Development of an instrument based on 10 Likertscales. Assessment and Evaluation in Higher Education, 32, 667-679. https://doi.org/10.1080/02602930601117191

Stern, M., Powell, R. \& Ardoin, N. (2008). What Difference Does It Make? Assessing Outcomes from Participation in a Residential Environmental Education Program. The Journal of Environmental Education, 39(4), 1-43. https://doi.org/10.3200/JOEE.39.4.31-43

Uttal, D., Meadow, N., Tipton, E., Hand, L., Alden, A., Warren, C. \& Newcombe, N. (2013). The malleability of spatial skills: A meta-analysis of training studies. Psychological Bulletin,

139(2),

352402. https://doi.org/10.1037/a0028446

Vilarrasa Cunillé, A. (2005). El medio local como escala de análisis en la didáctica de las ciencias sociales de educación secundaria. Enseñanza de las ciencias sociales, 4, 11-20. https://www.raco.cat/index.php/EnsenanzaCS/article/view/126211

Zabalza, M.A. (2006). La Universidad y la docencia en el mundo de hoy. Pontificia Universidad Javeriana. 